\title{
PROPUESTA DE UNA NUEVA METODOLOGÍA PARA DETERMINAR LA EFECTIVIDAD DE LOS DIQUES EN LA RETENCIÓN DE SEDIMENTOS
}

\author{
V. DÍAZ ${ }^{1}$, J. MONGIL ${ }^{1}$, J. NAVARRO ${ }^{2}$ \\ 'Grupo de Hidrología y Conservación, Universidad Católica de Ávila, \\ Canteros s/n 05005-Ávila, España. \\ ${ }^{2}$ Unidad de Hidráulica e Hidrología, Universidad de Valladolid, \\ Av. Madrid 44, 34004-Palencia, España.
}

RESUMEN. Los diques son estructuras transversales construidas en los cauces para mitigar la erosión, empleadas frecuentemente en España en proyectos de restauración hidrológico-forestal. Sus principales funciones son: controlar y retener los materiales sólidos transportados, estabilizar las laderas y los cauces torrenciales y reducir la velocidad del agua y consecuentemente su capacidad erosiva. No obstante, los efectos de los diques empleados en las restauraciones hidrológico-forestales para frenar la erosión son objeto de discusión en diversos estudios publicados en los últimos años. Con el fin de comprobar el grado de efectividad de los diques de gaviones en la retención de sedimentos se plantea una metodología basada en la estimación del volumen de la cuña mediante un levantamiento topográfico de detalle. Se ha comparado la metodología propuesta, que conlleva un exhaustivo y preciso trabajo de campo, con otras dos existentes, de uso frecuente y más simple elaboración. El procedimiento metodológico se ha aplicado a una cárcava situada en la cuenca del río Corneja, en Tórtoles (Ávila), donde se localiza una restauración hidrológico-forestal proyectada en 1964 y ejecutada en 1965. Se observa que los diques han conseguido retener un volumen de sedimentos de $237.95 \mathrm{~m}^{3}$, según la metodología planteada, lo que supone entre un 7.9 y un $30.3 \%$ superior al obtenido por las otras dos metodologías utilizadas. Los volúmenes unitarios de los diques, respecto a nuestra metodología, oscilan según el caso entre $\pm 5 \%$ y $\pm 50 \%$. Los resultados obtenidos con el método propuesto muestran una discrepancia con el resto, pero una mejor medición del volumen real de la cuña de sedimentos retenidos por los diques, lo cual justifica su empleo para la valoración de la efectividad de estas estructuras en el control de la erosión.

\section{Proposal of a new methodology to assess the effectiveness of check-dams}

ABSTRACT. Check dams are transverse structures that are built in some streams to mitigate erosion, and frequently used in forest-hydrology restoration projects in Spain. Their main functions are: controlling and retaining solid 
particles transported by flow discharge; slope and bed stream stabilization; reducing flow water velocities and their erosivity. Nevertheless, the erosion control effectiveness of check dams is a matter under discussion during the last years. Because of this, we want to verify how the check dams work controlling sediment yields, and so on how they reduce soil losses in gullies. We propose a method based in estimating the sediment volume retained through an accurate topographical survey. Our results have been compared with two other methods, most frequently used because of their geometric simplicity. A significant variation appears between the three methods. Therefore, to probe the effectiveness of the check dams a better estimation of their sediment retained surface justifies the full topographical survey. We have worked in Tórtoles (Ávila, Spain), in a deep gully from the Corneja river headwaters. There is a forest-hydrological restoration that was projected in 1964 and executed one year later. The topographical survey results show that check dams in the gully have retained $237.95 \mathrm{~m}^{3}$ of sediments. This is a $30.3 \%$ and a $7.9 \%$ bigger than the calculated by the other two methods. The unit volumes of the check dams range between $\pm 5 \%$ y $\pm 50 \%$ respect the obtained with our method. Our results show important differences compared with the other methodologies, but a better land surveying justifies the use of the topographic method to estimate the check dams effectiveness.

Palabras clave: restauración hidrológico-forestal, hidrotecnia, conservación de suelos, cárcavas, badlands, diques de retención.

Key words: forest-hydrological restoration, hydrotechnic, soil conservation, gullies restoration, badlands, check dams.

Enviado el 26 de diciembre de 2012 Aceptado el 14 de mayo de 2013

*Correspondencia: Grupo de Hidrología y Conservación. Universidad Católica de Ávila. Canteros s/n 05005-Ávila, España. E-mail: virginia.diaz@ucavila.es

\section{Introducción}

Un dique es una estructura transversal construida en un cauce con la intención de mitigar y reducir la erosión del lecho y laderas (Hudson, 1982; Morgan, 1997; Chanson, 2004; Zeng et al., 2009). La construcción de este tipo de estructuras ha sido una técnica empleada en los planes y programas de restauración hidrológico-forestal, en particular ampliamente utilizada en España y, principalmente, en el sureste de la Península Ibérica (Varela, 1999; Martínez-Lloris et al., 2001; Martínez de Azagra et al., 2002; Martín-Rosales et al., 2003; Belmonte et al., 2005a, 2005b; Boix-Fayos et al., 2007; Castillo et al., 2007, Romero et al., 2007; Conesa y García, 2009; Conesa et al., 2009, Castillo et al., 2011). En estas zonas semiáridas, en las que la degradación de la cubierta vegetal y la torrencialidad de las precipitaciones originan procesos erosivos que dañan gravemente al suelo, el empleo de esta técnica es especialmente importante y necesario. 
Las funciones que cumplen estas estructuras transversales y que justifican su empleo son múltiples, y han sido reseñadas por varios autores: controlan el transporte de sedimentos (Catella et al., 2005); estabilizan las laderas y cauces torrenciales, gracias a la cuña de materiales aguas arriba del dique, que se interpone entre las dos laderas, impidiendo los movimientos en masa, como deslizamientos de fondo, socavación o hundimientos (Conesa, 2004; Gil, 2004; Conesa y García, 2007; Romero, 2008); capturan, controlan, retienen y disminuyen el aporte de material sólido (Martín-Rosales et al., 2003; Conesa, 2004; Belmonte et al., 2005a, 2005b; Conesa y García, 2007; Romero, 2008); recargan los acuíferos, gracias al almacenamiento de agua en el vaso y el aumento de la carga hidrostática (Romero et al., 2003; Conesa, 2004; Gil, 2004; Conesa y García, 2007); disminuyen la velocidad del agua y por tanto su capacidad erosiva (Belmonte et al., 2005a, 2005b; Romero, 2008); retrasan el aterramiento de embalses y alargan su vida útil, al reducir la tasa de materiales que serían vertidos en el vaso del embalse (Gil, 2004; Belmonte et al., 2005a; Romero, 2008); y controlan aludes, mediante su retención completa o parcial, evitando que se vean afectadas vías de comunicación, poblaciones o estaciones de esquí (Gil, 2004).

Sin embargo, algunos autores consideran que los diques de retención de sedimentos no siempre resultan necesarios ni efectivos (Marston y Dolan, 1999; Romero, 2008); otros analizan los riesgos que entrañan estas obras de corrección, sobre todo en áreas especialmente vulnerables (García-Ruiz et al., 1996; Götz, 2001), e indican, como efectos negativos, que alteran la dinámica de los sedimentos y la estabilidad del lecho del cauce, interrumpen el movimiento longitudinal de nutrientes y organismos acuáticos, y obstruyen el paso de las olas de las inundaciones (Conesa, 2004; Wohl, 2006; BoixFayos et al., 2008). Es necesario el estudio de cada caso para determinar la necesidad de su utilización, y considerar si se pudiera lograr el efecto deseado únicamente mediante el empleo de vegetación. Castillo et al. (2011) concluyen que, a largo plazo, los usos del suelo ejercen un mayor control de la producción de sedimentos que las obras hidráulicas, que son menos eficientes con el aumento de la cobertura vegetal. No obstante, ambas actuaciones -cambios en los usos del suelo y construcción de obras hidráulicaspueden alterar el régimen hidrológico del cauce y su morfología, de forma que pueden llegar a anular los efectos beneficiosos conseguidos con la ejecución de estas obras (Boix-Fayos et al., 2007; Castillo et al., 2007, 2011). Por tanto, en el corto plazo, la construcción de diques tiene efectos positivos para el control de los sedimentos (MartínRosales et al., 2003), pero debe considerarse como una medida temporal, que debe ser acompañada con actuaciones de repoblación con especies fundamentalmente leñosas que ayuden a conseguir un control a largo plazo. De hecho, esta combinación de actuaciones tiene efectos positivos sobre la vegetación ribereña, de acuerdo con Bombino et al. (2006), que encuentran una diversificación longitudinal de los tipos de vegetación y la creación de nuevos hábitats para las comunidades biológicas y ecológicas. Así mismo, es necesario considerar la distinta repercusión de estas hidrotecnias en corrientes naturales y en zona de "badlands" o torrentes generados por una acción antrópica descontrolada. En este último caso, los diques intentan recuperar una morfología más parecida a la original antes de la intervención humana o al menos, si esto no fuera posible, disminuir la capacidad erosiva de los flujos torrenciales creados y reducir el elevado trans- 
porte de sedimentos, por lo que sus efectos serían positivos. Por otra parte, si se construyen estas hidrotecnias sobre flujos casi permanentes, se debe tener en cuenta a la fauna ictícola y, por lo tanto, adoptar las medidas oportunas para evitar la fragmentación del hábitat.

Por último, los efectos de la retención de sedimentos y laminación de avenidas anteriormente señalados, conducen a una reducción de los riesgos de inundación de los núcleos de población que se sitúan aguas abajo de estas estructuras (Gil, 2004), incrementando el tiempo de concentración. Sin embargo, en ocasiones inducen una falsa idea de seguridad absoluta y se ubican edificaciones u otros usos no adecuados en los vasos o conos de deyección.

Dado el problema de la erosión y, particularmente, de la erosión en cárcavas, y que el estudio de los efectos de los diques empleados en las restauraciones hidrológico-forestales para frenarla ofrece conclusiones diversas según autores, el objetivo de este trabajo es desarrollar una metodología para cuantificar el volumen real de sedimentos retenidos en los diques, mediante la medición precisa de la cuña de sedimentación, así como su aplicación a los diques de una cárcava situada en la restauración hidrológico-forestal de Tórtoles (Ávila). Así mismo, se realiza un análisis comparativo respecto a las metodologías de Castillo et al. (2007) y Romero (2008).

\section{Metodología}

La metodología propuesta estima el volumen de la cuña de sedimentos que han sido retenidos en los diques de corrección hidrológica. En primer lugar es preciso realizar un levantamiento topográfico de la cuña y de su asiento originario. A partir de estas mediciones, con el empleo de cálculos analíticos y caracterización de los sedimentos, se obtiene la masa real, en toneladas, de los sedimentos que conforman la cuña. Con estos datos y conociendo los diques proyectados, su antigüedad, y la superficie de la cuenca vertiente al dique, se consigue obtener la erosión que los diques han retenido en la zona de estudio. En los siguientes párrafos se expone en detalle el desarrollo metodológico.

\subsection{Estimación del lecho original}

Consiste en realizar un levantamiento topográfico (mediante estación total o dispositivo GPS de precisión centimétrica) a partir de dos mediciones. Se toma una primera medición para delimitar el extremo final de la cuña de sedimentos. Esta cuestión puede resultar compleja, y precisa una observación detallada de los sedimentos, y en concreto de la diferencia de tamaño o granulometría del material sedimentado. Si la cuña termina con una determinada anchura, hay que ajustar estas mediciones a la forma observada (Fig. 1). La segunda medición consiste en realizar una sección transversal inmediatamente aguas abajo del dique (Fig. 2), tomando con la estación total o GPS, un punto cada $0.5 \mathrm{~m}$. La estimación del lecho del cauce sobre el que se produjo la deposición de sedimentos tras la construcción del dique se consigue mediante la unión de cada uno de estos puntos con el punto o puntos tomados en la primera medición (Fig. 3). 


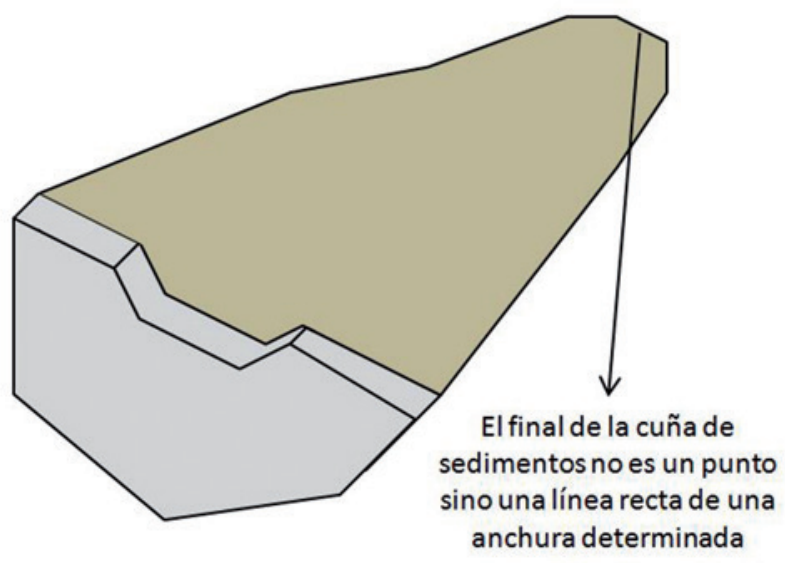

Figura 1. Cuña de sedimentos que no termina en un punto.

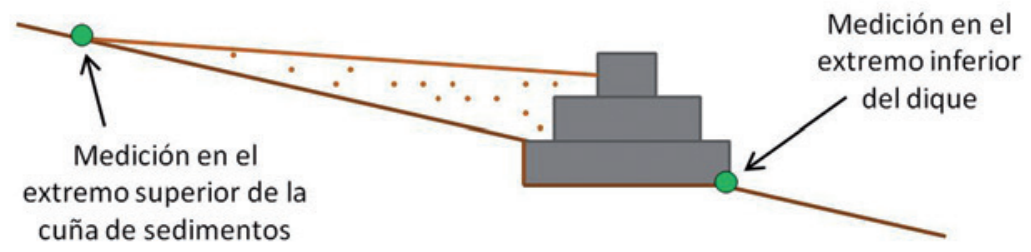

Figura 2. Mediciones a realizar, representadas en el perfil longitudinal de la cárcava.

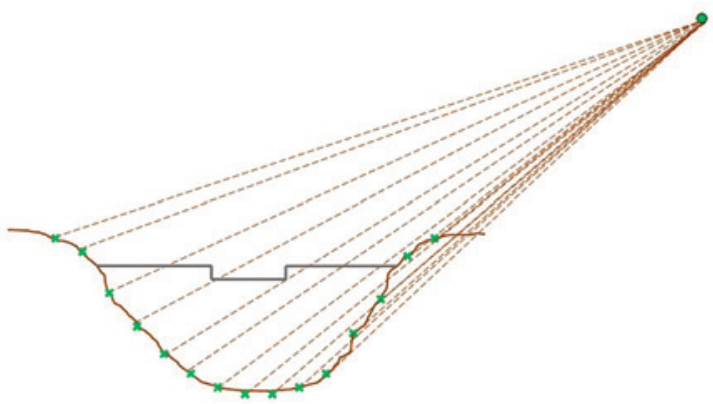

Figura 3. Resultado gráfico de la unión de las mediciones realizadas, obteniendo el lecho donde se asienta la cuña de sedimentos. 


\subsection{Medición de la cuña de sedimentos actual}

Se basa en realizar un levantamiento topográfico de la cuña de sedimentos a través de secciones transversales, para obtener su superficie actual. La distancia entre una sección transversal y la siguiente varía de 0.5 a $3 \mathrm{~m}$, en función de la longitud de la cuña. En cada sección se toma un punto cada $0.5 \mathrm{~m}$, y también se toman mediciones adicionales a cada extremo de la cuña, para obtener la sección transversal de las laderas (Figs. 4 y 5).

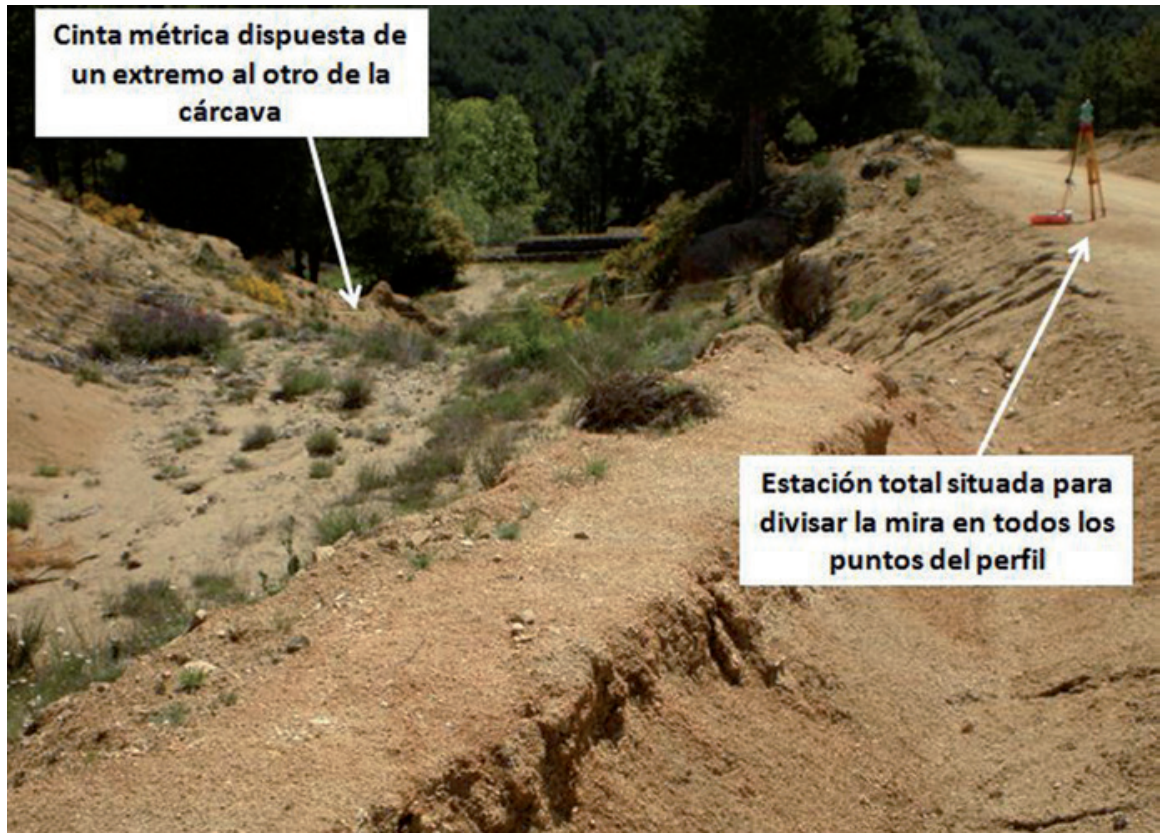

Figura 4. Disposición de la estación total y de la cinca métrica para comenzar a levantar topográficamente una sección de la cuña de sedimentos.

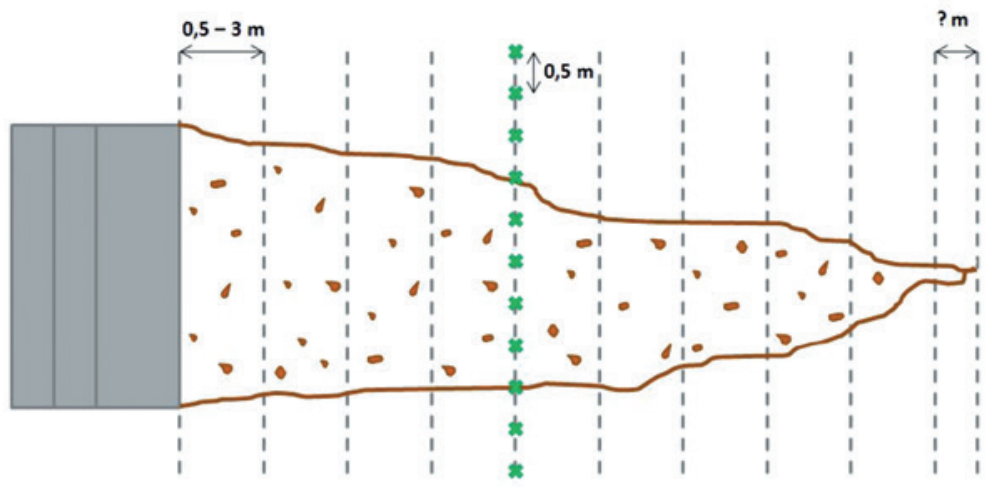

Figura 5. Vista en planta de las mediciones de las secciones transversales a la cuña de sedimentos. 


\subsection{Estimación del volumen de la cuña de sedimentación}

Para determinar el volumen de la cuña de sedimentos, expresado en $\mathrm{m}^{3}$, se determina el área de cada sección transversal de la cuña, unificando en cada sección los datos anteriores. Para ello se aplica una metodología de cálculo analítico a través de cálculo matricial. Conocidas las áreas de cada sección transversal de la cuña, y la distancia entre las dos secciones transversales, se puede calcular el volumen parcial de cuña entre cada par de secciones $\left(V_{p}\right)$, aplicando la fórmula del prismoide:

$$
V_{p}=\frac{h}{6} \cdot\left(B_{1}+B_{2}+4 \cdot B_{m}\right)
$$

donde $B_{1}$ y $B_{2}$ son las áreas de las secciones 1 y 2 , respectivamente (bases del prismoide), $B_{m}$ es el área de la base media del prismoide, y $h$ la distancia entre las bases del prismoide (Fig. 6). También se puede calcular el volumen parcial entre secciones empleando la fórmula de la media de las secciones extremas $\left(V_{M S E}\right)$ :

$$
V_{M S E}=\frac{B_{1}+B_{2}}{2} \cdot h
$$

donde $B_{1}$ y $B_{2}$ son las áreas de las secciones 1 y 2 , y $h$ la distancia entre las bases. Sin embargo, la fórmula de la media de las secciones extremas ofrece resultados poco exactos, ya que considera que el área transversal varía linealmente con la longitud, de forma que un análisis comparativo indica que el cálculo del volumen aplicando la fórmula del prismoide ofrece una mayor precisión (Casanova, 2002).

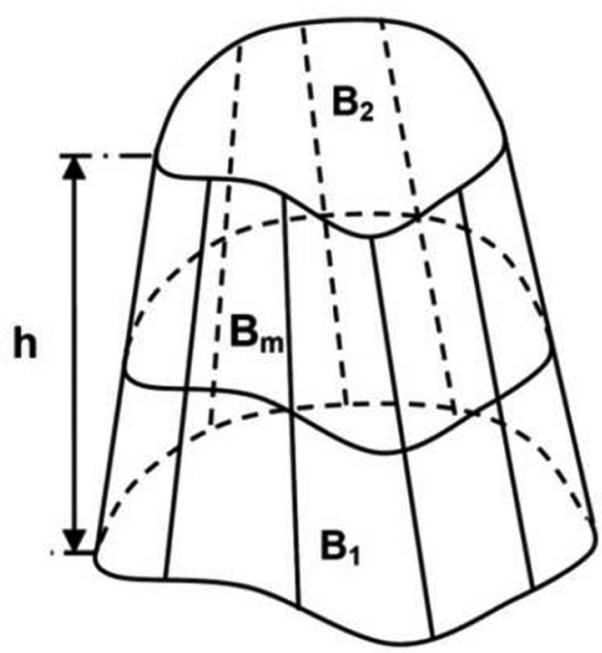

Figura 6. Cálculo de volúmenes entre secciones mediante la fórmula del prismoide. 
Por último, el volumen de tierras entre dos secciones transversales consecutivas, siempre que éstas sean homólogas, también se puede calcular aplicando la fórmula de la sección media $\left(V_{S M}\right)$, que asimila el sólido engendrado a un prisma de base la sección media $\left(B_{m}\right)$ :

$$
V_{S M}=B_{m} \cdot h
$$

donde $h$ es la distancia entre las bases del prisma. Esta fórmula precisa una sección intermedia, por lo que únicamente se puede emplear entre secciones alternas.

Cuando se requiere una mayor precisión en el cálculo del volumen se recomienda utilizar la fórmula del prismoide (Casanova, 2002). Al utilizar las otras dos ecuaciones planteadas (media de las secciones extremas y sección media) en lugar de la del prismoide, se comete un error, que se determina mediante la diferencia entre los valores obtenidos por las fórmulas de la media de las secciones extremas y de la sección media con el valor obtenido por la fórmula del prismoide. Se cumple que el error cometido al aplicar la fórmula de la sección media $\left(A_{l}\right)$ es la mitad y de signo contrario que el error cometido al utilizar la fórmula de la media de las secciones extre$\operatorname{mas}\left(A_{2}\right)$.

No obstante, hay que tener en cuenta la morfología del dique, ya que cuando una parte de éste quede aterrada, por ejemplo cuando el paramento aguas arriba es escalonado, se debe restar el volumen del dique que queda aterrado, ya que en caso contrario se estaría cometiendo un error de sobredimensionamiento del volumen final de sedimentos.

Por último, para determinar el volumen total de la cuña de sedimentos, se suman las cantidades obtenidas en el paso anterior.

\subsection{Estimación de la erosión}

Una vez determinado el volumen de sedimentos retenidos y la densidad aparente de los sedimentos, aplicando el método del cilindro, se obtiene el peso estimado de materiales retenidos en la cuña de sedimentación. Con estos datos, conociendo la fecha de construcción del dique y la superficie de aporte de sedimentos se estima el umbral mínimo de erosión $(E)$, en toneladas por hectárea y año, aplicando la ecuación:

$$
E=\frac{P}{T \cdot S}
$$

siendo $P$ el peso, en toneladas; $T$ el tiempo transcurrido desde la construcción de la hidrotecnia, medido en años; y $S$ la superficie en hectáreas de aporte de sedimentos.

\subsection{Metodologías planteadas por otros autores}

Otros autores como Castillo et al. (2007) y Boix-Fayos et al. (2008) asemejan el volumen de sedimentos retenido aguas arriba del dique a un prisma con sección rectangular, basándose en los estudios de Lien (2003) y May y Gresswell (2003), que consi- 
deran que las ramblas suelen formar cauces con sección transversal en U. Sin embargo, esta aproximación supone una forma geométrica que no se ajusta a la realidad en cauces diferentes a los estudiados por estos autores. Como datos toman únicamente la anchura, profundidad y longitud de la cuña. Castillo et al. (2007) señalan que, sin embargo, en zonas montañosas, y con pequeños arroyos tributarios, se observa una sección transversal con forma de V. En éstos, la anchura media de la cuña de sedimentos ( $w$ ) se estima como la media entre el ancho del canal en la sección aguas abajo del dique $\left(w_{b}\right)$, y la anchura media de la cuña de sedimentación $\left(w_{s}\right)$. El volumen de sedimentos almacenados aguas arriba de los diques se estima aplicando la fórmula:

$$
V=\frac{w \cdot l_{s} \cdot h}{2}
$$

donde $w$ es la anchura media del canal rellenado de sedimentos, $l_{s}$ es la longitud de la cuña de sedimentos, y $h$ es la altura de los sedimentos medidos desde la base del dique (Fig. 7).

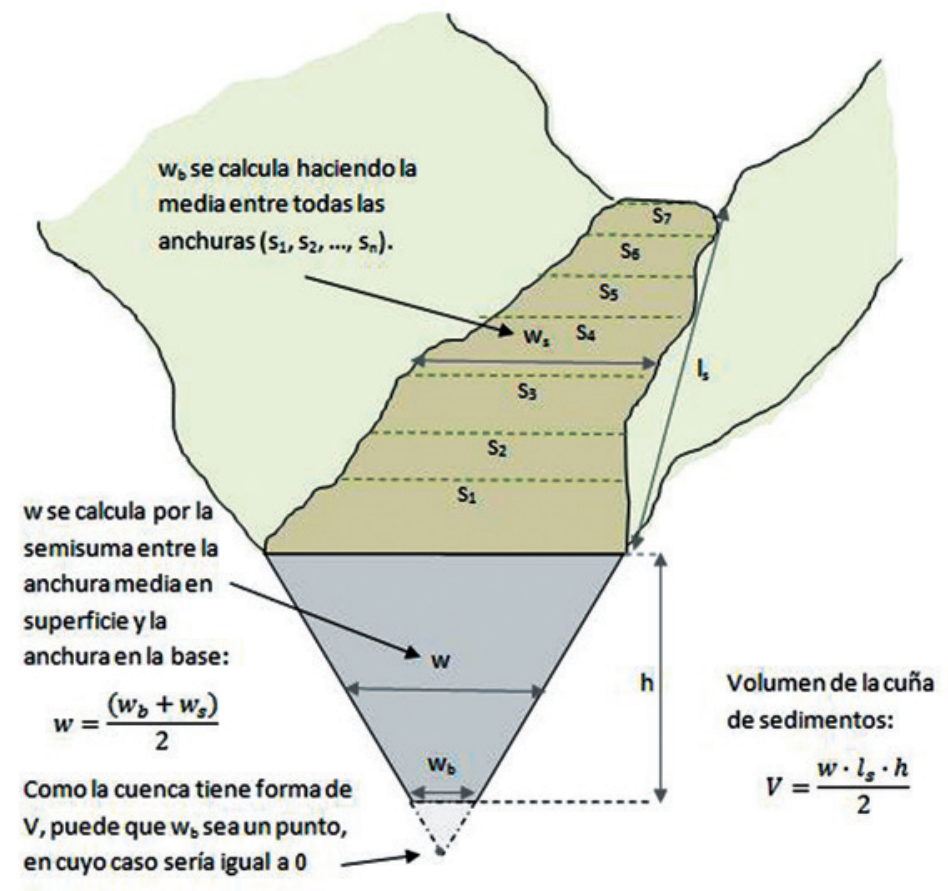

Figura 7. Cálculo del volumen (V) de la cuña de sedimentos siguiendo la metodología propuesta por Castillo et al. (2007). $\mathrm{S}_{\mathrm{i}}=$ anchura de la cuña en la sección transversal $i ; \mathrm{w}_{\mathrm{s}}=$ anchura media de la cuña de sedimentación (calculado como la media de las anchuras de la cuña en la secciones transversales $i$ ); $\mathrm{w}_{\mathrm{b}}=$ ancho de la base del dique en la sección aguas debajo de éste; $\mathrm{w}=$ anchura media del canal relleno de sedimentos; $\mathrm{h}=$ altura de los sedimentos medidos desde la base del dique $; 1_{\mathrm{s}}=$ longitud de la cuña de sedimentos. 
Por último, Romero (2008) asocia el volumen de la cuña de sedimentos con una pirámide de base trapezoidal en posición horizontal, figura geométrica que considera más parecida a la forma tridimensional de los sedimentos acumulados en el dique. De esta manera, la ecuación que utiliza para el cálculo del volumen de sedimentos en los diques es la del volumen de la pirámide de base trapezoidal $(V)$, delimitada por la superficie de los sedimentos sobre el dique:

$$
V=\frac{B \cdot H}{3}
$$

donde $B$ es el área de la base coincidente con el dique, y $H$ la longitud de la cuña de sedimentos (Fig. 8). Esta estimación supone que el extremo de la cuña termina en un punto, y no en todos los casos es así, de forma que también se simplifica bastante al generalizar el uso de esta fórmula para cualquier cuña. Por ello, se hace necesaria una metodología que mejore la precisión y se aproxime más a la realidad, mediante mediciones más detalladas y representativas.

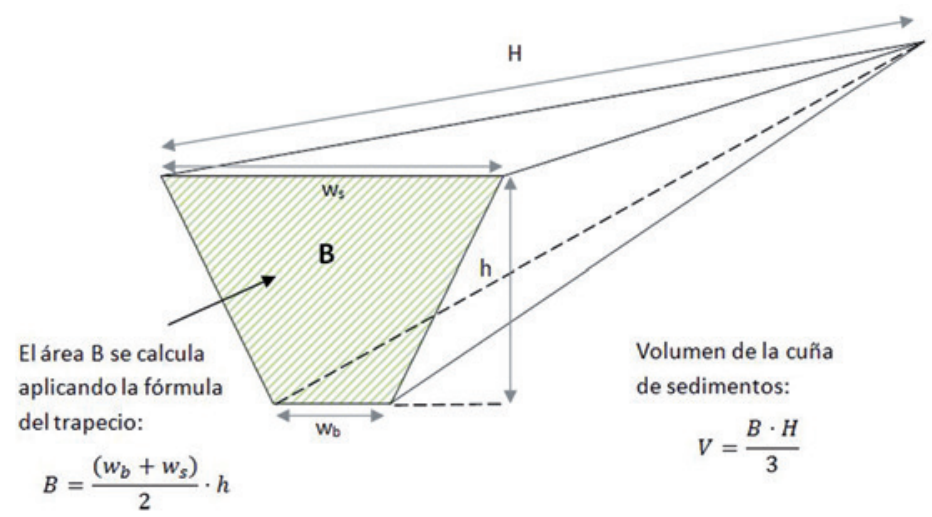

Figura 8. Cálculo del volumen (V) de la cuña de sedimentos siguiendo la metodología propuesta por Romero (2008). $\mathrm{w}_{\mathrm{s}}=$ ancho de la base del dique en la sección aguas arriba de éste; $\mathrm{w}_{\mathrm{b}}=$ ancho de la base del dique en la sección aguas debajo de éste; $\mathrm{h}=$ altura de los sedimentos medidos desde la base del dique; $\mathrm{B}=$ área de la base coincidente con el dique; $\mathrm{H}=$ longitud de la cuña de sedimentos.

\section{Metodología utilizada en este estudio}

\section{1. Área de estudio}

La zona de estudio se localiza en Tórtoles (Coordenadas UTM: 308 563, 4492 536; huso 30, datum ETRS89), municipio de la provincia de Ávila, situado en el Sistema Central, al sur de la Cuenca del Duero, subcuenca del Tormes, en pleno valle del río Corneja (Fig. 9). La precipitación anual media es de $571 \mathrm{~mm}$ y la temperatura media anual $10.6^{\circ} \mathrm{C}$. Los materiales geológicos que afloran son fundamentalmente plutónicos, tratándose en general de monzogranitos biotíticos porfídicos de grano grueso (IGME, 2008), que presentan un aspecto meteorizado y alterado, formando bolos y canchales. Los suelos resultantes son Orthents y Xerepts (USDA, 2010), con cerca de un $70 \%$ de 
arena, textura desde franco-arcillo-arenosa a arenosa y $\mathrm{pH}$ alrededor de 6. Las laderas y barrancos de Tórtoles estaban sometidas a un estado de degradación severo, causado principalmente por mantener las tierras sin arbolado para facilitar el pastoreo (Mongil et al., 2012). Dado su estado, esta zona fue objeto de un proyecto de restauración hidrológico-forestal redactado en 1964 (Azcarretazábal, 1964), que contemplaba la repoblación con diversas especies de pinos de 428.41 ha de cuencas de pequeños torrentes y arroyos tributarios del río Corneja, en altitudes comprendidas entre 1100 y 1500 m. La repoblación resultó exitosa, con espesuras medias actuales que rondan los 2800 pies ha-1. Así mismo se proyectó la construcción de 313 diques forestales de gaviones, de los que tras un exhaustivo inventario se han localizado 123, que se han clasificado en cuatro modelos, con alturas que varían entre 1 y $6 \mathrm{~m}$, y en los que se utilizaron gaviones de base, con una altura de $0.5 \mathrm{~m}$ (Martín-Vide, 2002). Gracias al menos en parte a estas actuaciones, el embalse de Santa Teresa, construido en 1960 y situado en el río Tormes a corta distancia de la desembocadura del Corneja, no ha visto mermada su capacidad por el aporte de sedimentos procedentes de los torrentes de esta zona.

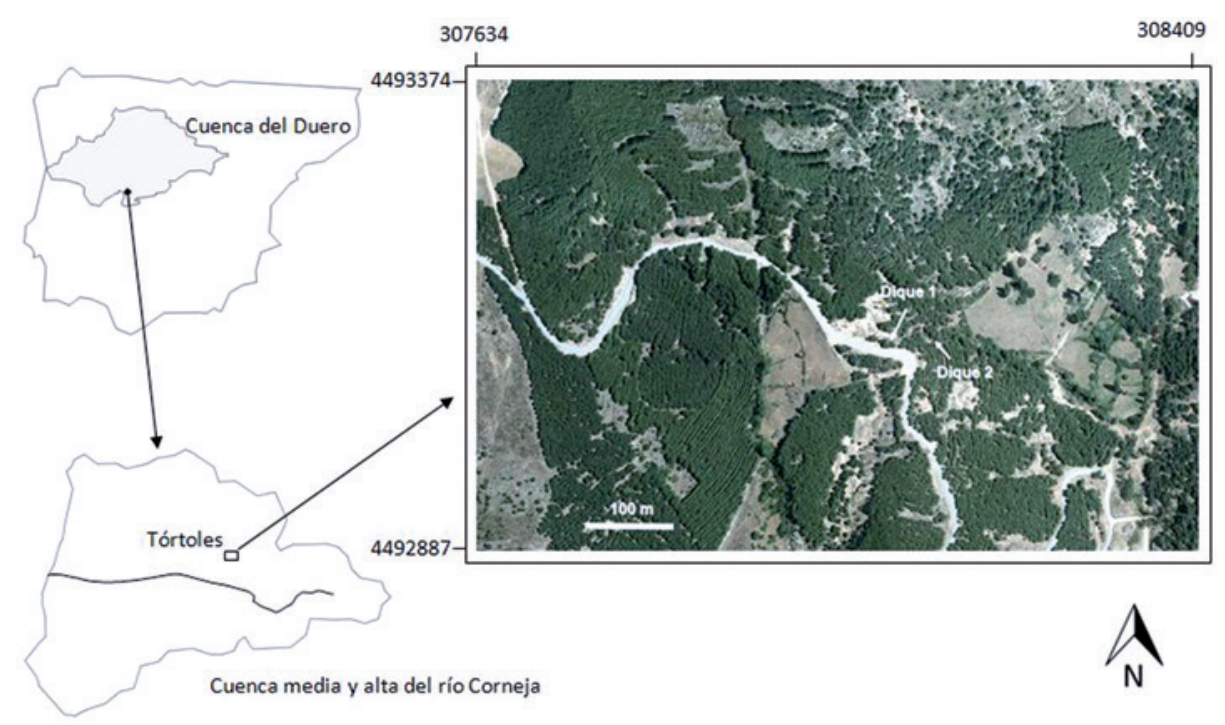

Figura 9. Localización del área de estudio y situación de los diques muestreados.

Este estudio se ha centrado en una cárcava de 109 m de longitud, $14 \mathrm{~m}$ de anchura media y $15 \%$ de pendiente longitudinal.

\subsection{Estimación del lecho original}

La metodología propuesta se ha aplicado a una cárcava en la que se construyeron dos diques de gaviones y se han medido las dos cuñas de sedimentos de la forma que se expone a continuación. El dique 1 es de $9.8 \mathrm{~m}$ de longitud y 7 pisos de gaviones, y el dique 2 es de $17.8 \mathrm{~m}$ de longitud y 8 pisos de gaviones; ambos con vertedero y gaviones de $0.5 \mathrm{~m}$ de altura. 


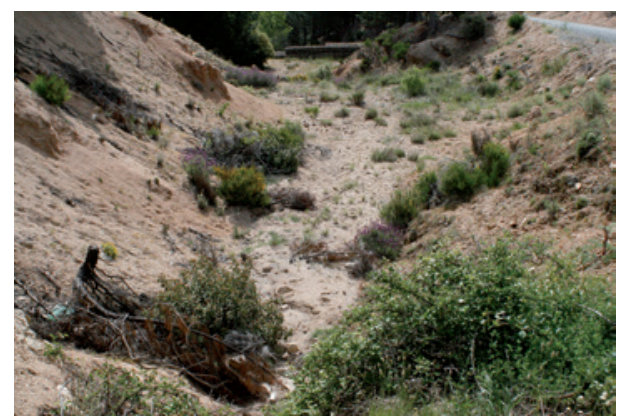

Figura 10. Cuña de sedimentos del dique 1.

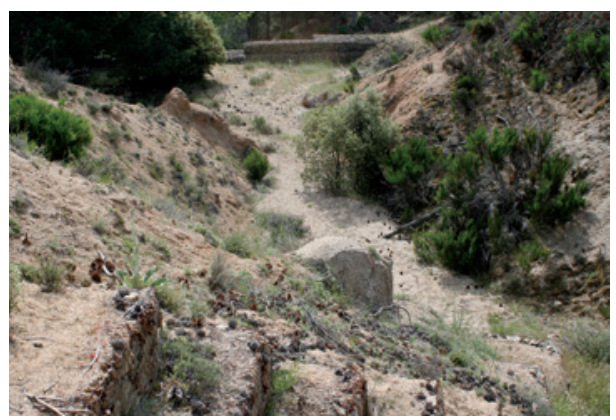

Figura 11. Cuña de sedimentos del dique 2.

Se ha estimado el lecho original sobre el que se asientan cada una de las dos cuñas existentes en la cárcava seleccionada, mediante la medición de sus extremos superiores, y de la sección transversal inmediatamente aguas abajo de cada dique, como definitoria del cauce antes de la construcción de los diques. En los dos casos, las cuñas terminan en un único punto final, como se puede observar en las Figs. 10 y 11.

\subsection{Medición de la cuña de sedimentos actual}

La cuña del dique 1 (el situado más aguas arriba), mide $28.08 \mathrm{~m}$ de longitud, tiene una anchura máxima de $9 \mathrm{~m}$ y una pendiente media del 17\%, y se ha medido a través de 10 secciones transversales, distanciadas $3 \mathrm{~m}$ entre sí, y el punto final, situado a $1.08 \mathrm{~m}$ de la sección 10. En cuanto a la cuña del dique 2 (situado aguas abajo del anterior), de $31.00 \mathrm{~m}$ de longitud, $12.00 \mathrm{~m}$ de anchura máxima y pendiente media del 14\%, se ha medido a través de 11 secciones transversales, distanciadas igualmente $3 \mathrm{~m}$, y el punto final está situado a $1 \mathrm{~m}$ de la sección 11 .

En cada una de las secciones transversales se han ido tomando puntos de derecha a izquierda, considerando que las secciones avanzan de aguas abajo a aguas arriba, hasta terminar en el punto final. Por este orden, se han tomado dos puntos iniciales correspondientes a la pendiente natural de las laderas colindantes a la cuña, a 2 y 1 m respectivamente del comienzo de ésta; a continuación se han tomado los puntos correspondientes a la cuña, distanciados $0.5 \mathrm{~m}$ entre sí, excepto el último punto, cuya distancia del anterior depende de la anchura de la cuña en cada sección; y por último, dos puntos correspondientes a las laderas en la margen izquierda de la cuña, distanciados 1 m entre sí y con respecto al último punto correspondiente a la cuña de sedimentos.

\subsection{Estimación del volumen de la cuña de sedimentación}

Con los datos obtenidos de la estimación del lecho original sobre el que se asienta la cuña de sedimentos, se han obtenido las cotas de los puntos coincidentes con cada una de las secciones transversales tomadas en la superficie de la cuña, de forma que para cada una de éstas se obtiene un polígono delimitado por el cauce estimado en su parte inferior, y por la superficie de la cuña en su parte superior. En ocasiones ha sido necesario realizar una estimación adicional para que el polígono quede cerrado, con ayuda 
de la pendiente obtenida de las laderas. Aplicando cálculo matricial se ha determinado el área de cada uno de estos polígonos correspondiente a cada sección transversal; por tanto, 10 polígonos del dique 1 y 11 polígonos del dique 2 .

A continuación se ha determinado el volumen entre cada par de secciones aplicando la fórmula del prismoide, la fórmula de la media de las secciones extremas, y la fórmula de la sección media. En este último caso utilizando secciones alternas para disponer de una sección intermedia con el que aplicar la fórmula. Además, se ha determinado el error cometido en cada uno de los diques en la aplicación de las fórmulas, tomando como referencia la fórmula del prismoide. De acuerdo con Casanova (2002), se ha considerado que la fórmula del prismoide ofrece el valor más preciso.

Por último se han sumado, en cada dique, los volúmenes entre secciones obtenidos en la aplicación de la fórmula del prismoide, para obtener el volumen total de cada cuña de sedimentos.

\subsection{Estimación de la erosión}

Se ha estimado la densidad aparente de los sedimentos retenidos en cada hidrotecnia utilizando el método del cilindro, tomando dos muestras por cuña. Aplicando la densidad aparente al volumen de la cuña de sedimentos se obtiene la masa total de los materiales sedimentados en cada dique.

Por otra parte, se han consultado los años transcurridos desde la construcción del dique, que se corresponden con los años durante los cuales se ha producido la retención de sedimentos, mediante la consulta del proyecto ejecutado (Azcarretazábal, 1964). Por último, se ha estimado la superficie de aportación de sedimentos a cada hidrotecnia mediante la delimitación de la divisoria sobre el plano topográfico a escala 1:5000 y con el apoyo de la ortofotografía aérea. La superficie de aportación conjunta para ambos diques es de 1.02 ha. Con este dato, se ha estimado la cantidad de sedimentos retenida por los diques, en tha-1 año $^{-1}$.

\subsection{Comparación del resultado obtenido con otras metodologías}

Se han comparado los resultados obtenidos aplicando el método expuesto, y los obtenidos con las fórmulas de Castillo et al. (2007), considerando un perfil del cauce en forma de V, y de Romero (2008), que asemeja la cuña de sedimentos a una pirámide trapezoidal en posición horizontal.

\section{Resultados y discusión}

Siguiendo la metodología propuesta, se ha estimado el lecho original partiendo de la sección transversal inmediatamente aguas abajo de cada dique (Fig. 12) y el extremo superior de cada cuña. Con esta estimación y con la superficie de la cuña levantada en cada una de las secciones transversales, se ha medido el área encerrada por cada una de ellas mediante ajustes gráficos siguiendo las pendientes reales o, en su defecto, estimadas de las laderas del cauce (Fig. 13). A través de cálculo analítico se ha obtenido el área 
superficial de cada una de las secciones transversales en ambos diques (Tabla 1). Posteriormente se han aplicado las fórmulas del prismoide, de la sección media y de la media de las secciones extremas, para determinar el volumen total de sedimentos retenidos. Los errores $A_{1}$ y $A_{2}$, obtenidos de la aplicación de las fórmulas señaladas han sido muy bajos en ambos casos (Tabla 2). No obstante, es necesario restar del volumen total estimado el perteneciente a la parte del dique que se encuentra aterrado bajo la cuña de sedimentos. El volumen obtenido mediante la metodología propuesta (en adelante caso A) es $135.92 \mathrm{~m}^{3}$ de sedimentos retenidos por el dique 1 y $102.03 \mathrm{~m}^{3}$ por el dique 2 . Una vez obtenido el volumen de sedimentos retenidos, y utilizando la densidad aparente media de los sedimentos de la cuña (1.49 $\mathrm{t} \mathrm{m}^{-3}$ en la cuña 1 y $1.42 \mathrm{t} \mathrm{m}^{-3}$ en la cuña 2$)$, se obtiene que la masa estimada de sedimentos retenidos en la cuña es $202.24 \mathrm{t}$ en el caso del dique 1 y 144.41 t en el caso del dique 2 (Tabla 2). Esta masa de sedimentos se ha ido depositando desde que se construyeron los diques, de forma que el periodo de sedimentación hasta la toma de datos en el levantamiento topográfico ha sido de 46 años. De esta manera se obtiene una tasa de erosión media en la cárcava para la metodología propuesta (caso A) de $7.39 \mathrm{t} \mathrm{ha}^{-1}$ año $^{-1}$ (Tabla 2).
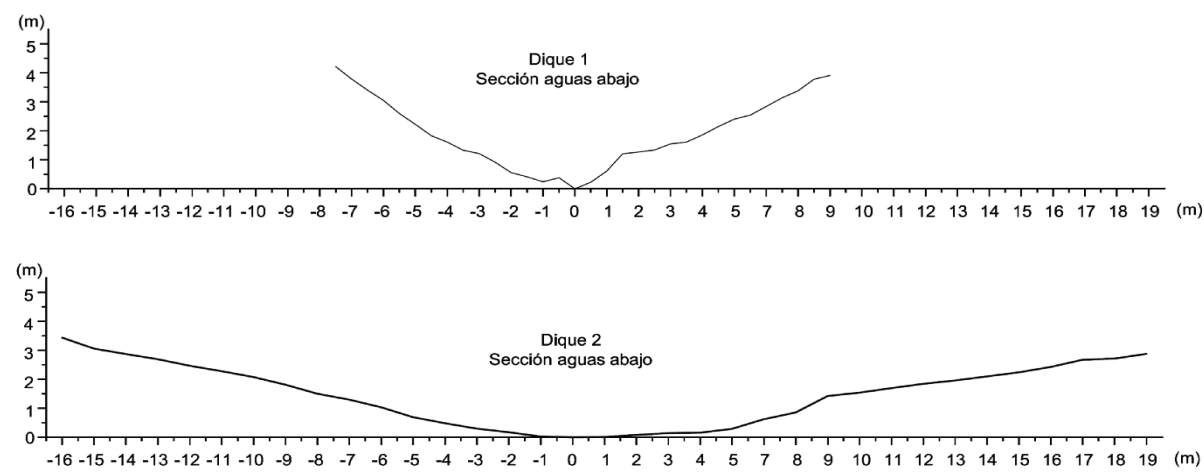

Figura 12. Sección transversal inmediatamente aguas abajo del dique 1 (arriba) y del dique 2 (abajo), a partir de las cuales se estiman los cauces originales.

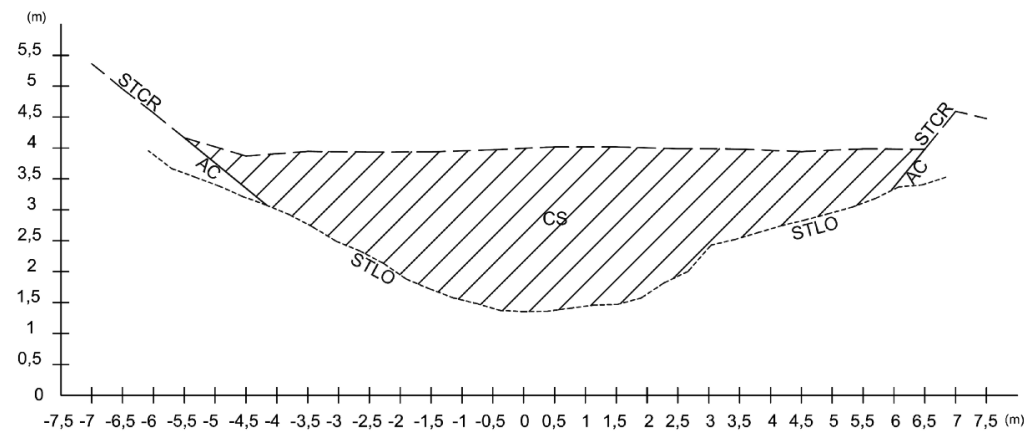

Figura 13. Sección transversal 1 del dique 2. STCR=sección transversal del cauce real; $C S=$ cuña de sedimentos; $A C=$ ajustes del cauce; $S T L O=$ sección transversal del lecho original . 
Tabla 1. Área superficial $\left(\mathrm{m}^{2}\right)$ de las secciones transversales de la cuñas de sedimentos retenida por los diques 1 y 2 .

\begin{tabular}{|c|c|c|c|}
\hline \multicolumn{2}{|c|}{ DIQUE 1 } & \multicolumn{2}{c|}{ DIQUE 2 } \\
\hline Sección & Área $\left(\mathbf{m}^{2}\right)$ & Sección & Área $\left(\mathbf{m}^{2}\right)$ \\
\hline 1 & 15.7560 & 1 & 18.9883 \\
\hline 2 & 17.2871 & 2 & 15.4948 \\
\hline 3 & 12.0941 & 3 & 12.2943 \\
\hline 4 & 6.9608 & 4 & 9.3101 \\
\hline 5 & 6.7444 & 5 & 6.4574 \\
\hline 6 & 5.1621 & 6 & 4.1691 \\
\hline 7 & 3.0442 & 7 & 2.5895 \\
\hline 8 & 1.9827 & 8 & 0.9464 \\
\hline 9 & 0.9725 & 9 & 0.9727 \\
\hline 10 & 0.3111 & 10 & 0.4291 \\
\hline- & - & 11 & 0.1830 \\
\hline
\end{tabular}

Tabla 2. Aplicación de las fórmulas del prismoide $\left(V_{P}\right)$, de la media de las secciones extremas $\left(V_{M S E}\right)$ y de la sección media $\left(V_{S M}\right)$ para obtener el volumen de sedimentos, errores obtenidos de su aplicación, pisos de gaviones y volumen de dique aterrados, volumen final de la cuña de sedimentos retenidos por los diques, densidad aparente media $(\mathrm{Da})$, masa de sedimentos $\left(M_{S}\right)$ y erosión cuantificada para ambos diques (caso A).

\begin{tabular}{|c|c|c|}
\hline & DIQUE 1 & DIQUE 2 \\
\hline $\mathbf{V}_{\mathbf{P}}\left(\mathbf{m}^{3}\right)$ & 187.37 & 186.03 \\
\hline $\mathbf{V}_{\text {MSE }}\left(\mathbf{m}^{3}\right)$ & 186.85 & 186.84 \\
\hline $\mathbf{V}_{S M}\left(\mathbf{m}^{3}\right)$ & 188.42 & 184.40 \\
\hline Error $A_{1}(\%)$ & -0.28 & 0.44 \\
\hline Error $A_{2}(\%)$ & 0.56 & -0.88 \\
\hline $\mathbf{N}^{0}$ PISOS DE GAVIONES ATERRADOS & 6 & 7 \\
\hline V $_{\text {DIQUE ATERRADO }}\left(\mathbf{m}^{3}\right)$ & 51.45 & 84.00 \\
\hline $\mathbf{V}_{\mathbf{C U N \tilde { A }}}\left(\mathbf{m}^{3}\right)$ & 135.92 & 102.03 \\
\hline Da $\left(t \cdot m^{-3}\right)$ & 1.4879 & 1.4154 \\
\hline $\mathbf{M}_{S}(\mathbf{t})$ & 202.24 & 144.41 \\
\hline Erosión $\left(t \cdot h a^{-1} \cdot a \tilde{a} \mathbf{o}^{-1}\right)$ & 7.39 & \\
\hline
\end{tabular}

Aplicando la metodología de Castillo et al. (2007) (en adelante caso B) (Tabla 3), se obtiene un volumen de $120.15 \mathrm{~m}^{3}$ en el dique 1 y $181.20 \mathrm{~m}^{3}$ en el dique 2 , que tras descontar en cada caso la parte del dique aterrada bajo la superficie de la cuña de sedimentos, resulta un volumen de $68.70 \mathrm{~m}^{3}$ y $97.20 \mathrm{~m}^{3}$, respectivamente. Con este volumen, la tasa de erosión (caso B) es de 5,11 t ha- $\mathrm{año}^{-1}$. 
Tabla 3. Parámetros empleados y resultados obtenidos de la aplicación de la metodología propuesta por Castillo et al. (2007) (caso B).

\begin{tabular}{|c|c|c|}
\hline Ancho & DIQUE 1 & DIQUE 2 \\
\hline$S_{1}$ & 9.00 & 12.00 \\
\hline$S_{2}$ & 9.80 & 10.98 \\
\hline$S_{3}$ & 8.50 & 11.50 \\
\hline$S_{4}$ & 6.38 & 9.50 \\
\hline$S_{5}$ & 6.10 & 7.50 \\
\hline$S_{6}$ & 5.25 & 7.50 \\
\hline$S_{7}$ & 4.29 & 4.00 \\
\hline$S_{8}$ & 3.20 & 2.00 \\
\hline$S_{9}$ & 2.61 & 3.50 \\
\hline$S_{10}$ & 1.92 & 3.00 \\
\hline$S_{11}$ & - & 2.00 \\
\hline
\end{tabular}

\begin{tabular}{|c|r|r|}
\hline & DIQUE 1 & DIQUE 2 \\
\hline$w_{s}$ & 5.71 & 6.68 \\
\hline$w_{b}$ & 0.00 & 0.00 \\
\hline$w$ & 2.85 & 3.34 \\
\hline$h$ & 3.00 & 3.50 \\
\hline$l_{s}$ & 28.08 & 31.00 \\
\hline$V_{\boldsymbol{B}}$ & $\mathbf{1 2 0 . 1 5}$ & $\mathbf{1 8 1 . 2 0}$ \\
\hline
\end{tabular}

$S_{i}=$ anchura de la cuña en la sección transversal $i ; w_{s}=$ anchura media de la cuña de sedimentación (calculado como la media de las anchuras de la cuña en la secciones transversales i); $w_{b}=$ ancho de la base del dique en la sección aguas debajo de éste; $w=$ anchura media del canal relleno de sedimentos; $h=$ altura de los sedimentos medidos desde la base del dique; $l_{s}=$ longitud de la cuña de sedimentos; $V_{B}=$ volumen de la cuña de sedimentos obtenido de la aplicación de la fórmula de Castillo et al. (2007).

Al aplicar la metodología propuesta por Romero (2008) (en adelante caso C), con los valores de los parámetros que se muestran en la tabla 4, se obtiene un volumen de $137.59 \mathrm{~m}^{3}$ en el dique 1 y $217.00 \mathrm{~m}^{3}$ en el dique 2 . A estos valores se descuenta la parte del dique aterrada bajo la superficie de la cuña de sedimentos, resultando un volumen de $86.14 \mathrm{~m}^{3}$ y $133 \mathrm{~m}^{3}$, respectivamente. Con este volumen, la tasa de erosión (caso C) es de $6.74 \mathrm{t} \mathrm{ha}^{-1} \mathrm{año}^{-1}$.

Tabla 4. Parámetros empleados y resultados obtenidos de la aplicación de la metodología propuesta por Romero (2008) (caso C).

\begin{tabular}{|c|c|c|}
\hline & DIQUE 1 & DIQUE 2 \\
\hline$w_{s}$ & 9.80 & 12.00 \\
\hline$w_{b}$ & 0.00 & 0.00 \\
\hline$h$ & 3.00 & 3.50 \\
\hline$B$ & 14.70 & 21.00 \\
\hline$H$ & 28.08 & 31.00 \\
\hline$V_{C}$ & $\mathbf{1 3 7 . 5 9}$ & $\mathbf{2 1 7 . 0 0}$ \\
\hline
\end{tabular}

$w_{s}=$ ancho de la base del dique en la sección aguas arriba de éste; $w_{b}=$ ancho de la base del dique en la sección aguas debajo de éste; $h=$ altura de los sedimentos medidos desde la base del dique; $B$ =área de la base coincidente con el dique; $H=$ longitud de la cuña de sedimentos; $V_{C}=$ volumen de sedimentos obtenido de la aplicación de la fórmula de Romero (2008) 
Tabla 5. Tiempo empleado en trabajo de campo, volumen y masa de la cuña de sedimentos, y valores de erosión para ambos diques, obtenidos de la aplicación de las distintas metodologías.

\begin{tabular}{|l|c|c|r|r|r|c|}
\hline \multirow{2}{*}{} & \multicolumn{2}{|c|}{ CASO A } & \multicolumn{2}{c|}{ CASO B } & \multicolumn{2}{c|}{ CASO C } \\
\cline { 2 - 7 } & DIQUE 1 & DIQUE 2 & DIQUE 1 & DIQUE 2 & DIQUE 1 & DIQUE 2 \\
\hline TIEMPO $(\mathrm{h})$ & 3.00 & 3.25 & 1.63 & 1.75 & 0.94 & 1.00 \\
\hline VOLUMEN $\left(\mathrm{m}^{3}\right)$ & 135.92 & 102.03 & 68.70 & 97.20 & 86.14 & 133.00 \\
\hline MASA (t) & 202.24 & 144.41 & 102.22 & 137.58 & 128.17 & 188.25 \\
\hline EROSIÓN $\left(\mathrm{t} \cdot \mathrm{ha}^{-1} \cdot\right.$ año $\left.^{-1}\right)$ & \multicolumn{2}{|c|}{7.39} & \multicolumn{3}{|c|}{5.11} & \multicolumn{2}{c|}{6.74} \\
\hline
\end{tabular}

En la tabla 5 se realiza una comparación de resultados para cada dique y en cada uno de los casos indicados (A, B y C), incluyendo el tiempo empleado para su aplicación. En el caso A (metodología propuesta), se ha estimado una media de 15 minutos por sección transversal más 15 minutos dedicados a estacionamiento y colocación de cinta métrica, y en los casos $\mathrm{B}$ y $\mathrm{C}$, los tiempos estimados dependen del número de parámetros que es necesario medir en campo, y se ha considerado un tiempo medio de 5 minutos por parámetro.

El tiempo empleado (Tabla 5 y Fig. 14) es notablemente superior en el caso A que en los otros dos casos, concretamente un $46 \%$ más que el caso B y un $69 \%$ más que el caso C. Se trata por lo tanto de un inconveniente para la utilización de la metodología propuesta, salvo que la precisión justificara un mayor coste en tiempo y dinero. Aunque en los tres métodos se estima la forma de la cuña de sedimentos en su parte más profunda, que apoya sobre el antiguo lecho, a este respecto la metodología propuesta es geométricamente más ajustada que las otras dos, y contempla un mayor número de mediciones de campo que, además, son más detalladas y representativas. Así mismo, la metodología propuesta estima el lecho original del cauce (cárcava o barranco) partiendo la proyección de la sección de aguas abajo del dique, proporcionalmente hacia aguas arriba, en conjunción con la proyección de las laderas según su pendiente actual. Esta estimación resulta más aproximada que una proyección puramente geométrica, en concreto un canal prismático de sección rectangular (Castillo et al., 2007) y una pirámide de base trapezoidal (Romero, 2008), ya que intenta acomodarse al perfil natural del terreno. El equipo de medición empleado también es importante, aunque las metodologías en sí no varían. Así, una estación total o un GPS de precisión centimétrica ofrecen valores de suficiente exactitud, mientras que un GPS con errores superiores al metro no es recomendable.

El cálculo del volumen de sedimentos (Tabla 5) ofrece resultados diferentes dependiendo de la metodología y del dique medido. Haciendo un análisis para cada dique (Fig. 15), el volumen de sedimentos retenidos por el dique 1 es un $49.5 \%$ mayor en el caso A que en el caso B y un $36.6 \%$ mayor que en el caso C; y para el dique 2, el volumen es en el caso A un $4.7 \%$ mayor que en el caso B y un $30.4 \%$ menor que en el caso C. Considerando conjuntamente los dos diques, se ha conseguido retener un volumen de tierra de $237.95 \mathrm{~m}^{3}$, según la metodología planteada, lo que muestra la importancia de 
esas obras en el control de la erosión, por lo que se considera necesario replantearse los resultados de métodos más simples. Este valor supone un $30.3 \%$ y un $7.9 \%$ superior, respectivamente, al obtenido por las otras dos metodologías estudiadas. Al contabilizar el volumen en conjunto de los dos diques se compensan las diferencias entre las metodologías, dado que estas diferencias son positivas en unos casos y negativas en otros y eso parece inducir que los resultados finales de las diferentes metodologías son comparables, aunque realmente no sea así.

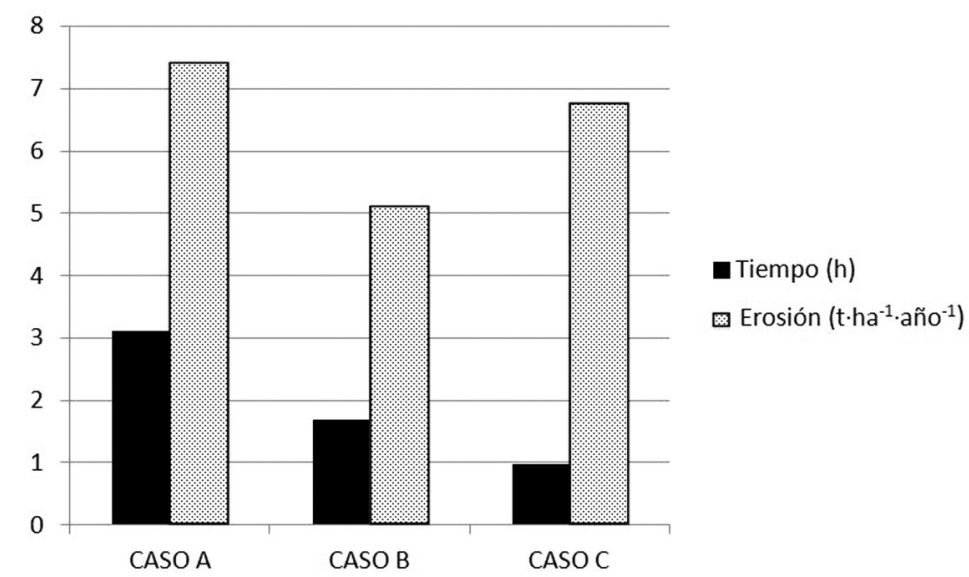

Figura 14. Erosión obtenida mediante las distintas metodologías (casos A, B y C), y tiempo empleado en aplicarlas.

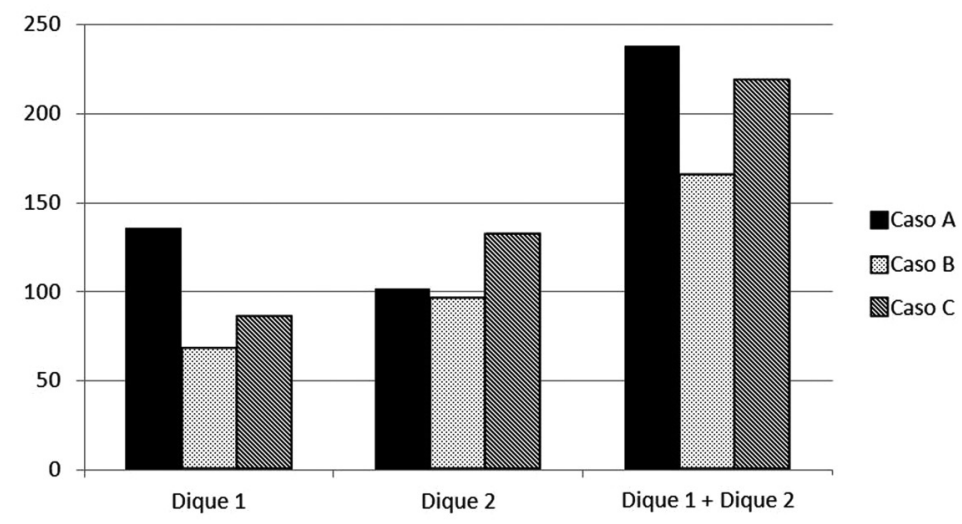

Figura 15. Volumen de sedimentos retenidos $\left(\mathrm{m}^{3}\right)$, según las diferentes metodologías. 
Si se observan los resultados de erosión ( $\mathrm{t} \mathrm{ha}^{-1} \mathrm{año}^{-1}$ ) (Tabla 5 y Fig. 14), expresados en valores conjuntos para ambos diques, las estimaciones son similares para las tres metodologías comparadas, con una diferencia entre el mayor valor (caso A) y el menor (caso B) de $2.28 \mathrm{t} \mathrm{ha}^{-1} \mathrm{año}^{-1}$. El resultado obtenido por la metodología propuesta es superior a las otras dos, más próximo a la metodología de Romero (2008). A este respecto hay que señalar lo dicho ya para los volúmenes, y es que al analizar los resultados conjuntos de erosión para ambos diques, las diferencias que son de distinto signo se compensan, por lo que puede darse una falsa sensación de que los resultados de las tres metodologías son comparables.

Finalmente hay que decir que las cifras obtenidas de erosión media son sólo un umbral mínimo, pues los diques no retienen la totalidad de los sólidos en suspensión, por lo que una buena parte de los limos y las arcillas se han escapado fuera de la cuenca. Fifield (2004) indica que la eficiencia de las estructuras de retención de sedimentos en obras con movimiento de tierras es siempre menor del 100\%; Makusic (2007) ha obtenido con trampas de sedimentos una eficiencia creciente del 37\% al 99\% según es mayor el periodo de retorno usado para su diseño. Sin embargo, la porosidad de los gaviones hace que las tasas habituales de retención de sedimentos aplicados no sirvan de referencia. Martín-Rosales et al. (2003) han obtenido un coeficiente de retención del $35 \%$ en diques de gaviones en la Sierra de Gádor con diversos episodios de lluvia. Romero et al. (2012), empleando la fórmula de "trap efficiency" de Brown (1943), han obtenido valores que varían entre $42.3 \%$ y $83.9 \%$ de eficiencia en la retención de sedimentos, con una tasa media de sedimentos de $1.7 \mathrm{t} \mathrm{ha}^{-1}$ año ${ }^{-1}$, para 18 diques de gaviones y de hormigón construidos en la Cuenca del río Quípar (Murcia) en los años 1962 y 1996. Por otra parte, se ha tomado como referencia temporal el periodo de 46 años que va desde la ejecución de la restauración (1965) hasta la fecha en que se hicieron las mediciones (marzo de 2011); sin embargo, es posible que, aunque no se ha encontrado referencia documental al respecto, la ejecución del proyecto se retrasara, o que las cuñas de sedimentos se terminaran de formar hace años, es decir, que los diques ya estuvieran aterrados antes de medir las cuñas. En cualquiera de estos casos el tiempo de cálculo se reduciría, con lo que los valores de erosión media aumentarían.

\section{Conclusiones}

Se propone una metodología que, a la vista de los resultados, se considera adecuada para medir la cuña de sedimentación retenida en los diques de corrección hidrológica, y de esta forma cuantificar sus efectos en la retención de la erosión. No obstante, la metodología sólo se ha aplicado a una cárcava en la que se sitúan dos diques, por lo que los resultados obtenidos deben tomarse con cautela, siendo necesario aplicar la metodología a un mayor número de diques, trabajo que se está realizando en este momento. Sin embargo, se observa que el tiempo empleado en aplicar la metodología propuesta es bastante más elevado que en otras metodologías, y esto puede ser un inconveniente en función de los objetivos perseguidos. Este trabajo muestra que las metodologías más sencillas pueden ser empleadas como primeras aproximaciones, pero dadas las diferencias que muestran incluso entre ellas mismas, no pueden utilizarse para argumentar o con- 
traargumentar la eficacia de las obras transversales. Para ello es necesario establecer una metodología suficientemente aproximada a la realidad del proceso a cuantificar. La metodología propuesta recaba un mayor número de datos de campo reales sobre la naturaleza superficial de las cuñas que otras metodologías, por lo que el volumen de sedimentos obtenido se aproxima más al volumen real de sedimentos retenidos en la cuña, ya que se cuenta con un elemento geométrico de cálculo medido de manera precisa. Por consiguiente, es de esperar un menor error que en metodologías menos detalladas y, por tanto, una mejor interpretación del papel de estas estructuras transversales.

El volumen de sedimentos obtenido de la aplicación del método planteado a una cárcava concreta permite estimar valores de erosión moderados (según la clasificación de FAO-PNUMA-UNESCO, 1980) respecto a las tasas tolerables para terrenos agrícolas, aunque son elevadas para las tolerables en la formación de suelos; pero estos datos son una aproximación al umbral mínimo de erosión, ya que los diques no son capaces de retener la totalidad de sedimentos removidos de una cuenca y no existe información sobre el momento exacto de su aterramiento.

\section{Agradecimientos}

Este trabajo ha sido parcialmente financiado por el proyecto de investigación P0001/2011 del convenio UCAV-Caja de Ávila. Los autores agradecen a la Dra. Eunice Maia de Andrade (Universidade Federal de Ceará, Brasil) la revisión del original.

\section{Referencias}

Azcarretazábal, D. 1964. Proyecto de repoblación forestal y restauración de laderas en la cuenca río Corneja. Tramo I. Término municipal de Tórtoles. Confederación Hidrográfica del Duero, Ministerio de Obras Públicas.

Belmonte, F., Romero, A., Martínez-Lloris, M. 2005a. Erosión en cauces afectados por obras de corrección hidrológica (Cuenca del Río Quípar, Murcia). Papeles de Geografía 41-42, 71-83.

Belmonte, F., Romero, A., Martínez-Lloris, M. 2005b. Impactos ambientales asociados a la construcción de obras de corrección hidrológica en la Cuenca del río Quipar (Sureste de España): apertura de caminos de acceso. Cuadernos de Investigación Geográfica 31, 97-106.

Boix-Fayos, C., González, G., López-Bermúdez, F., Castillo, V. 2007. Effects of check dams, reforestation and land-use changes on river channel morphology: case study of The Rogativa Catchment (Murcia, Spain). Geomorphology 91, 103-123.

Boix-Fayos, C., de Vente, J., Martínez-Mena, M., Barberá, G.G., Castillo, V. 2008. The impact of land use change and check-dams on catchment sediment yield. Hydrological Processes 22, 4922-4935.

Bombino, G., Tamburino, V., Zimbone, S.M. 2006. Assessment of the effects of check-dams on riparian vegetation in the mediterranean environment: a methodological approach and example application. Ecological Engineering 27, 134-144.

Brown, C.B. 1943. Discussion of sedimentation in reservoirs. En Proceedings of the American Society of Civil Engineers 69, J. Witzig (ed.), American Society of Civil Engineers, pp. 1493-1500. 
Casanova, L. 2002. Topografía Plana. Universidad de los Andes, Facultad de Ingeniería, Departamento de vías, Venezuela.

Castillo, V., Mosch, W., Conesa, C., González, G., Navarro Cano, J.A., López-Bermúdez, F. 2007. Effectiveness and geomorphological impacts of check dams for soil erosion control in a semiarid mediterranean catchment: El Cárcavo (Murcia, Spain). Catena 70, 416-427.

Castillo, V., Boix-Fayos, C., de Vente, J., Martínez-Mena, M., González, G. 2011. Efectos del aumento de la cubierta vegetal y los diques de corrección en la producción de sedimentos de cuencas mediterráneas. Cuadernos de la Sociedad Española de Ciencias Forestales 32, 153 158.

Castella, M., Paris, E., Solari, L. 2005. Case study: Efficiency of slit-check dams in the Mountain region of Versilia Basin. Journal of Hydraulic Engineering, 145-152.

Chanson, H. 2004. The hydraulics of open cannel flow: an introduction. Elsevier ButterworthHeinemann, Oxford, $495 \mathrm{pp}$.

Conesa, C. 2004. Los diques de retención en cuencas de régimen torrencial: diseño, tipos y funciones. Nimbus 13-14, 125-132.

Conesa, C., García, R. 2007. Litofacies de relleno y modelo de sedimentación de los diques de retención en el tramo inferior de la Rambla del Cárcavo (Cuenca del Segura). Cuaternario y Geomorfología 21 (3-4), 77-100.

Conesa, C., García, R. 2009. Local scour estimation at check dams in torrential streams in south east Spain. Geografiska Annaler 91 A (3), 159-177.

Conesa, C., Arana, R., García, R. 2009. Variación granulométrica y mineralógica en profundidad de los sedimentos retenidos por diques. Estudio en cauces torrenciales semiáridos del sureste peninsular. Nimbus 23-24, 61-88.

FAO-PNUMA-UNESCO 1980. Metodología provisional para la degradación de los suelos. Roma.

Fifield, J. S. 2004. Designing for Effective Sediment and Erosion Control on Construction Sites. Forester Communications Inc., Santa Barbara, 290 pp.

García-Ruiz, J.M., White, S., Martí, C., Valero, B., Errea, M.P., Gómez-Villar, A. 1996. La catástrofe del Barranco de Arás (Biescas, Pirineo Aragonés) y su contexto espacio-temporal. Instituto Pirenaico de Ecología, Consejo Superior de Investigaciones Científicas, Zaragoza, 54 pp.

Gil, R. (Coord.) 2004. Restauración hidrológico-forestal: Pasado, presente y futuro. VII Congreso Nacional de Medio Ambiente, Madrid, 73 pp.

Götz, A. 2001. Flood Protection: A Common Goal for Federal Cantonal and Municipal Authorities. Federal Office for Water and Geology.

Hudson, N. 1982. Conservación del suelo. Reverté, Barcelona, 350 pp.

IGME 2008. Mapa Geológico de España. Escala 1:50.000. Hoja 529: Santa María del Berrocal. Instituto Geológico y Minero de España, Madrid.

Lien, H.P. 2003. Design of slit dams for controlling stony debris flow. International Journal of Sediment Research 18, 74-87.

Makusic, M.S. 2007. Effects of Design Changes on Sediment Retention Basin Efficiency, NC State Theses and Dissertations. Disponible en: http://www.lib.ncsu.edu/resolver/1840.16/1938.

Marston, R.A., Dolan, L.S. 1999. Effectiveness of sediment control structures relative to spatial pattern of upland soil loss in an arid watershed, Wyoming. Geomorphology 31, 313-323.

Martín-Rosales, W., Pulido-Bosch, A., Gisbert, J., Vallejos, A. 2003. Sediment yield estimation and check dams in a semiarid area (Sierra de Gádor, southern Spain). En Erosion Prediction in Ungauged Basins: Integrating Methods and Techniques, IAHS Publ. 279, 51-58.

Martín-Vide, J.P. 2002. Ingeniería de ríos. Edicions UPC, Badalona, 404 pp.

Martínez de Azagra, A., Fernández, R., Seseña, A., Méndez, C., Díez, J.M., Navarro, J., Varela, J.M. 2002. Metodología para la inventariación de diques forestales gavionados. Aplicación 
en la provincia de Palencia. Cuadernos de la Sociedad Española de Ciencias Forestales 13, 171-181.

Martínez-Lloris, M., Romero, A., Alonso, F. 2001. Respuesta erosiva de cuencas, corregidas mediante diques de retención de sedimentos, ante lluvias de alta intensidad. Cuenca del río Quípar, sureste de España. Papeles de Geografía 34, 191-203.

May, C., Gresswell, R. 2003. Processes and rates of sediment and wood accumulation in headwater streams of the Oregon coast range, USA. Earth Surface Processes Landforms 28, 409424.

Mongil, J., Navarro, J., Cruz, V., Díaz, V. 2012. Destrucción y restauración de la cubierta forestal en la cuenca alta del río Corneja (Ávila): análisis histórico de un proceso de gestión cambiante. En Reunión del Grupo de Historia Forestal de la Sociedad Española de Ciencias Forestales, Vitoria.

Morgan, R.P.C. 1997. Erosión y conservación del suelo. Ediciones Mundi-Prensa, Madrid, 343 pp. Romero, A. 2008. Los diques de corrección hidrológica como instrumentos de cuantificación de la erosión. Cuadernos de Investigación Geográfica 34, 83-99.

Romero, A., Martínez-Lloris, M., Belmonte, F., Hernández, E. 2003. La Cuenca del Río Quipar (región de Murcia): Una cuenca modelo para el estudio de utilidad de los diques de retención de sedimentos en el control de la erosión y en la recarga de acuíferos. Nimbus 11-12, 89-105.

Romero, A., Alonso, F., Martínez-Lloris, M. 2007. Erosion rates obtained from check-dam sedimentation (SE Spain). A multi-method comparison. Catena 71, 172-178.

Romero, A., Marín-Sanleandro, P., Ortiz-Silla, R. 2012. Loss of soil fertility estimated from sediment trapped in check dams. South-eastern Spain. Catena 99, 42-53.

USDA 2010. Keys to Soil Taxonomy. Disponible en: http://soils.usda.gov/technical/classification Fecha de acceso: 02/9/2012.

Varela, J.M. (Dir) 1999. Inventario de obras de corrección hidrológica-forestal de las variables ambientales relacionadas con las mismas. Centro de Estudios de Técnicas Aplicadas, Dirección General de Obras Hidráulicas.

Wohl, E. 2006. Human impacts to mountain streams. Geomorphology 79, 217-248.

Zeng, Q.L., Yue, Z.Q., Yang, Z.F., Zhang, X.J. 2009. A case study of long-term field performance of check-dams in mitigation of soil erosion in Jiangjia Stream, China. Environmental Geo$\log y 58,897-911$. 